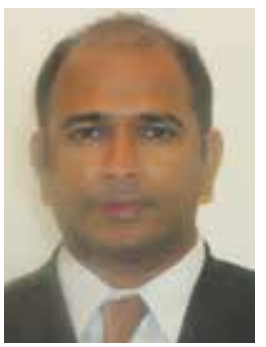

\title{
GIANT ANEURYSM OF THE ASCENDING AORTA
}

Venkateshwar Polsani, M.D. ${ }^{a}$; Angela M. Wright, M.D.b; Kamil Muhyieddeen, M.D.b; Faisal Nabi, M.D.'; Dipan J. Shah, M.D. ${ }^{a}$

${ }^{a}$ Methodist DeBakey Heart and Vascular Institute, The Methodist Hospital, Houston, TX; ${ }^{b}$ The Methodist Hospital, Houston, $T X$

V. Polsani, M.D.

\section{History}

A 28-year-old man with no prior medical history presented with new onset dyspnea. He was not on any medication at the time of admission, and he denied using cigarettes or alcohol. There is no family history of sudden cardiac death. A month prior to the admission he was running three miles a day. On physical examination he was 75 inches tall and weighed 163 lbs. His vital signs on admission were stable, although his cardiac examination revealed a grade III/VI early diastolic murmur.

The patient underwent an echocardiogram that showed a dilated left ventricle, moderate to severe aortic valve insufficiency, and dilated aortic root. A cardiovascular magnetic resonance (CMR) imaging study was ordered to assess for the severity of aortic valve insufficiency (AI) and the extent of aortic root dilation. The steady-state free precision Figures 1-6 shows a severely dilated left ventricle (LVEDD $9.2 \mathrm{~mm}$, LVEDV $505 \mathrm{~mL}$ ) and an aortic insufficiency jet. The volume-rendered three-dimensional image (Figure 7) shows a severely dilated aortic root $(7.2 \mathrm{~cm})$ and ascending aorta $(9.2 \mathrm{~cm})$. Image 8 shows the volumetric assessment of the aortic insufficiency (regurgitant volume $150 \mathrm{~mL}$, regurgitant fraction $75 \%$ ), which is categorized, as severe.

The patient underwent surgical resection of the aortic valve, aortic root, and ascending aorta. A 31-mm St. Jude conduit valve
Figure 1.
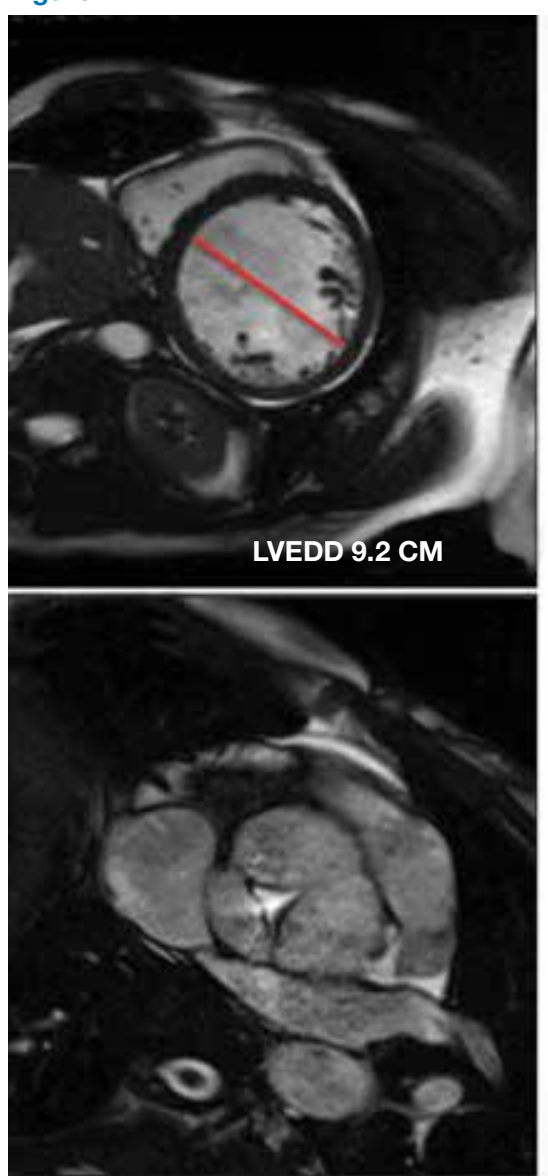

Figure 4.
Figure 2.
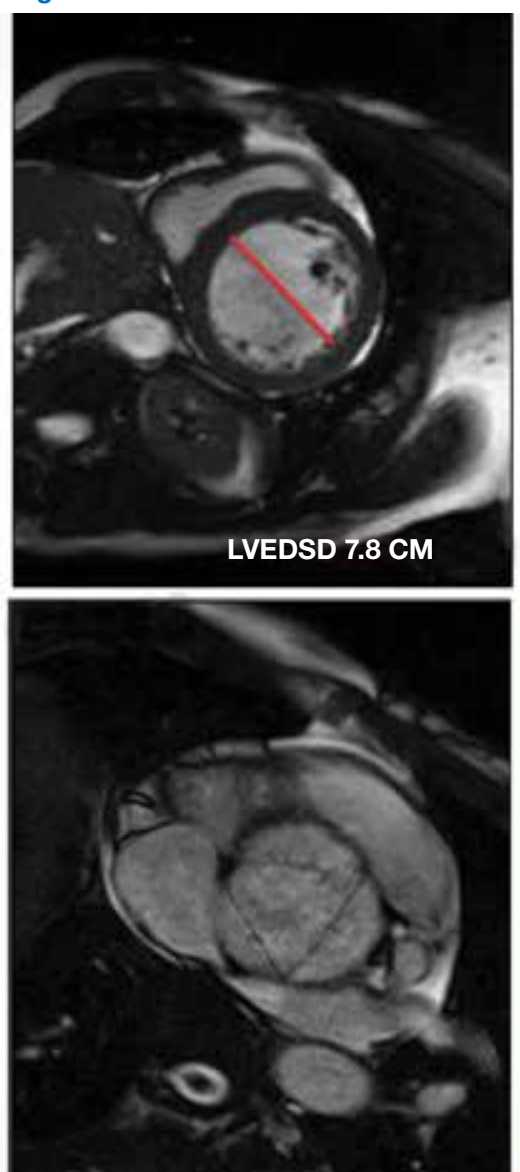

Figure 5.
Figure 3.
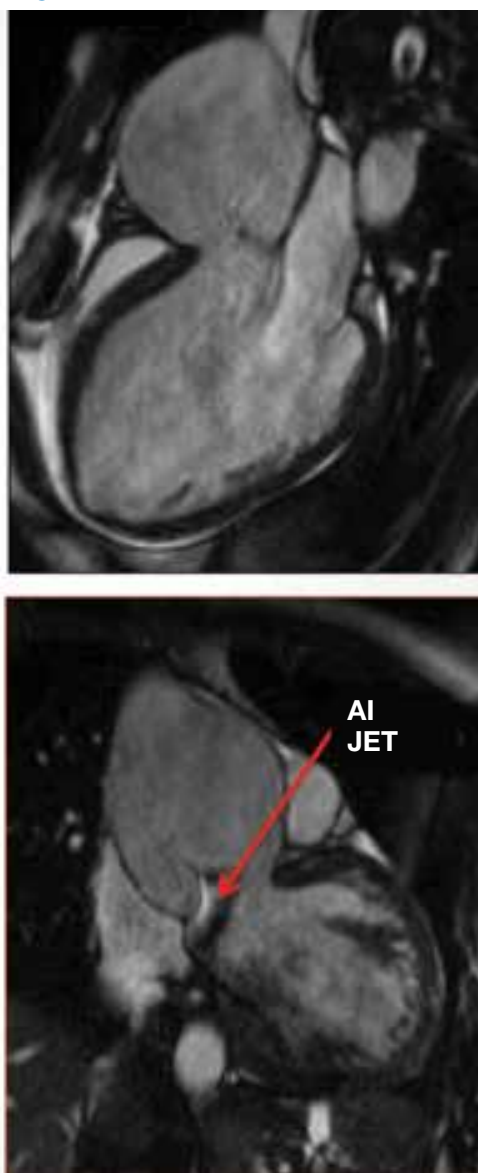

Figure 6. 
was used for the aortic valve replacement, and the coronary arteries were reimplanted on to the conduit. The patient did well during the postoperative course and was discharged home in stable condition.

The aortic valve and aortic wall were sent for surgical pathology. Figures 9 and 10 are of a hematoxylin and eosin (H\&E) stained slide showing myxoid degeneration of the aortic valve. Figure 12 is an H\&E stain of the aortic wall showing moderate fibrointimal proliferation, extensive medial degeneration with diffuse mucopolysaccharide deposition, and focal medial necrosis. These areas also have marked loss of the elastic fibers as shown on the elastin stain in Figure 11. The patient was diagnosed with Marfan syndrome based on the clinical and pathological findings.

\section{Discussion}

Marfan syndrome is an autosomal dominant connective tissue disorder with high penetrance but variable prevalence. ${ }^{1}$ About
$25 \%$ of cases are from sporadic mutation. ${ }^{1}$ Marfan syndrome results from mutations in the FBN1 gene. The FBN1 gene encodes fibrillin-1 glycoprotein that forms the extracellular matrix in the form of microfibrils. ${ }^{1}$ These microfibrils are found in the media of the ascending aorta and other elastin-containing tissues. In patients with Marfan syndrome, there is degeneration of elastin tissue and replacement of microfibrils in the media of the aorta with mucopolysccharides (myxoid degeneration).

Marfan syndrome involves the cardiovascular, ocular, and skeletal systems. ${ }^{3}$ Cardiovascular manifestations include thoracic aortic aneurysm/dissection, aortic insufficiency from the aortic root distortion, and the mitral insufficiency from the mitral valve prolapsed. ${ }^{2}$ The most feared complication of Marfan syndrome is a type A dissection. The differential diagnosis for Marfan syndrome includes Loeys-Dietz syndrome and Ehlers-Danlos syndrome ${ }^{3}$.

The current American College of Cardiology/American Heart Association (ACC/AHA) guidelines recommend annual imaging for
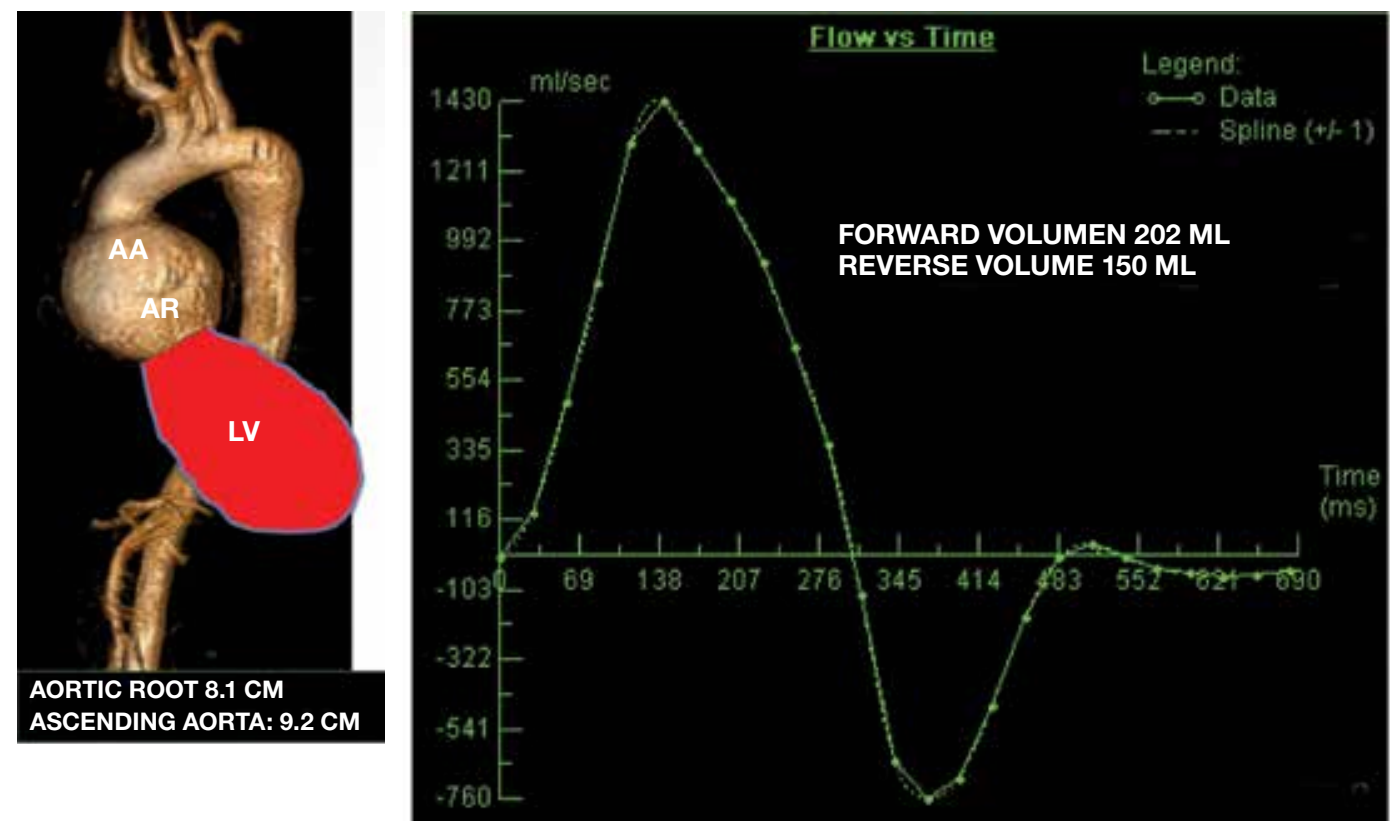

Figures 7-8.

Figures 9-10.

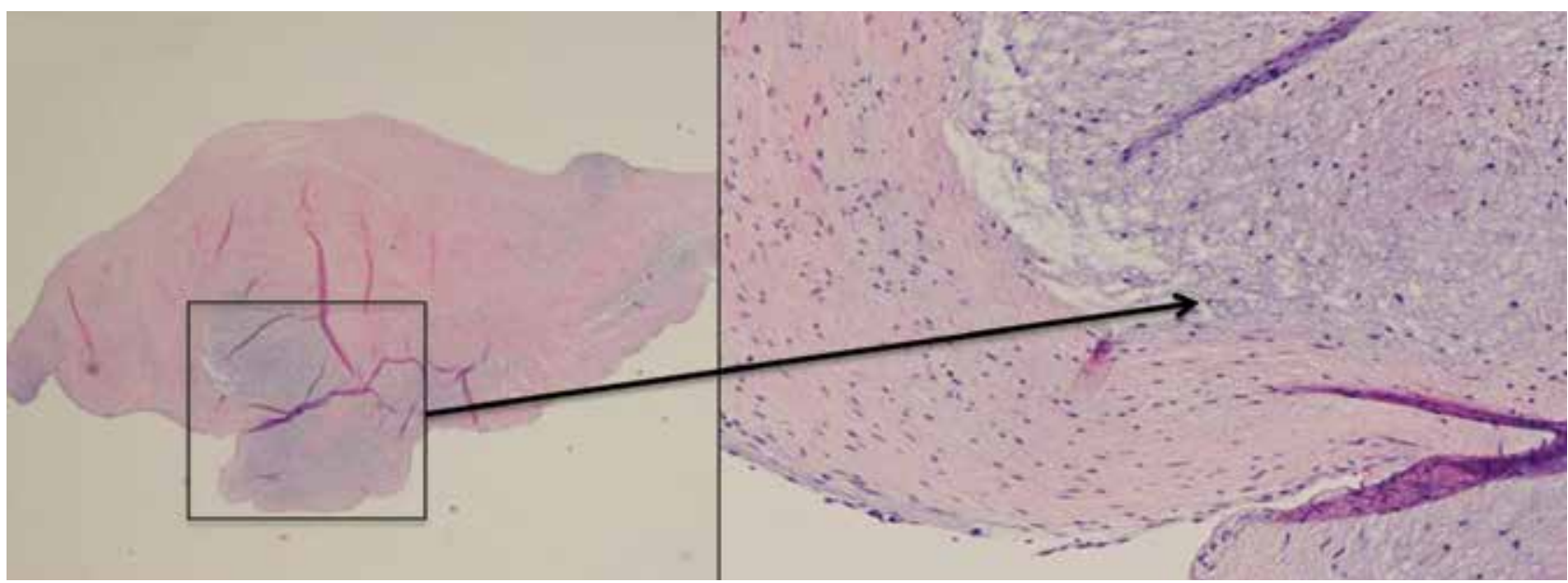



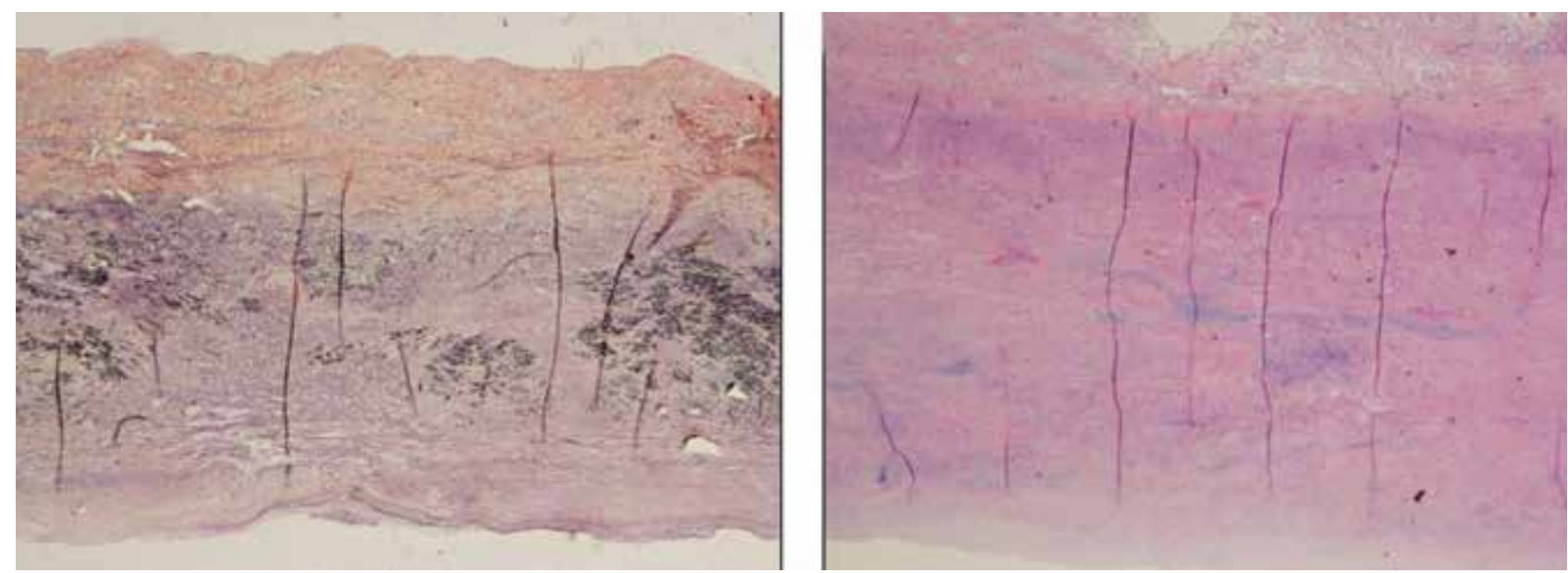

Figures 11-12.

patients with Marfan syndrome if the stability of aortic diameter is documented (Class I indication). ${ }^{3}$ If the maximum diameter is greater than $4.5 \mathrm{~cm}$, more frequent imaging (every 6 months) should be performed. The ACC/AHA guidelines recommend aortic imaging of first-degree relatives in patients with familial aortic aneurysms (class I). ${ }^{3}$ If one or more first-degree relatives have thoracic aortic aneurysm, then imaging of second-degree relatives is reasonable (class IIa). ${ }^{3}$ The guidelines also recommend surgical repair of the dilated aortic root/ascending aorta in patients with Marfan syndrome at $5.0 \mathrm{~cm}$ (external diameter measured by CT or MRI). ${ }^{2,3}$ The factors that would lead to surgical repair at a diameter less than $5 \mathrm{~cm}$ include rapid aneurysm growth $(>0.5$ $\mathrm{cm} /$ year), significant aortic insufficiency, or a family history of dissection at diameter $<5 \mathrm{~cm} .^{2,3}$

Our patient had an aortic diameter $>5 \mathrm{~cm}$ and had severe aortic insufficiency; he therefore underwent resection of the aortic root/ ascending aorta and the aortic valve. A 31-mm St. Jude conduit valve was placed with reimplantation of coronary arteries.

Conflict of Interest Disclosure: All authors have completed and submitted the Methodist DeBakey Cardiovascular Journal Conflict of Interest Statement and none were reported.

Funding/Support: The authors have no funding disclosures.

Keywords: Marfan syndrome, FBN1 gene, aneurysm, aortic insufficiency

\section{References}

1. LeMaire SA, Russell L. Epidemiology of thoracic aortic aneurysm. Nat Rev Cardiol. 2011 Feb;8(2):103-13.

2. Loeys BL, Dietz HC, Braverman AC, Callewaert BL, De Backer J, Devereux RB, et al. The revised Ghent nosology for the Marfan syndrome. J Med Genet. 2010 Jul;47(7):47685.

3. Hiratzka LF, Bakris GL, Beckman JA, Bersin RM, Carr VF, Casey DE Jr, et al. 2010 ACCF/AHA/AATS/ACR/ASA/SCA/SCAI/SIR/ STS/SVM Guidelines for the diagnosis and management of patients with thoracic aortic disease. A Report of the American College of Cardiology Foundation/American Heart Association Task Force on Practice Guidelines, American Association for Thoracic Surgery, American College of Radiology, American Stroke Association, Society of Cardiovascular Anesthesiologists, Society for Cardiovascular Angiography and Interventions, Society of Interventional Radiology, Society of Thoracic Surgeons, and Society for Vascular Medicine. J Am Coll Cardiol. 2010 Apr 6;55(14):e27-e129. 